\title{
Crosstalk in dynamic optical interconnects in photorefractive crystals
}

\author{
Andersen, Peter E.; Petersen, Paul Michael; Buchhave, Preben
}

Published in:

Applied Physics Letters

Link to article, DOI:

10.1063/1.112368

Publication date:

1994

Document Version

Publisher's PDF, also known as Version of record

Link back to DTU Orbit

Citation (APA):

Andersen, P. E., Petersen, P. M., \& Buchhave, P. (1994). Crosstalk in dynamic optical interconnects in photorefractive crystals. Applied Physics Letters, 65(3), 271-273. https://doi.org/10.1063/1.112368

\section{General rights}

Copyright and moral rights for the publications made accessible in the public portal are retained by the authors and/or other copyright owners and it is a condition of accessing publications that users recognise and abide by the legal requirements associated with these rights.

- Users may download and print one copy of any publication from the public portal for the purpose of private study or research.

- You may not further distribute the material or use it for any profit-making activity or commercial gain

- You may freely distribute the URL identifying the publication in the public portal

If you believe that this document breaches copyright please contact us providing details, and we will remove access to the work immediately and investigate your claim. 


\title{
Crosstalk in dynamic optical interconnects in photorefractive crystals
}

\author{
Peter E. Andersen \\ The Optics Group, Physics Department, Bldg. 309, The Technical University of Denmark, \\ DK-2800 Lyngby, Denmark \\ Paul M. Petersen \\ Optics and Fluid Dynamics Department, P. O. Box 49, DK-4000 Roskilde, Denmark

\begin{abstract}
Preben Buchhave
The Optics Group, Physics Department, Bldg. 309, The Technical University of Denmark, DK-2800 Lyngby, Denmark
\end{abstract}

(Received 7 February 1994; accepted for publication 6 May 1994)

\begin{abstract}
We have investigated the crosstalk between two neighboring gratings in photorefractive $\mathrm{Bi}_{12} \mathrm{SiO}_{20}$ optical interconnects. The gratings are induced by the interference between one reference beam and two object beams. By applying a suitable phase shift in one of the object beams, we can selectively switch off one of the gratings. The crosstalk between the two gratings is experimentally determined from the diffraction efficiency in the remaining grating before and after applying the phase shift. The magnitude of the crosstalk is determined by the intensity ratio between the reference beam intensity and the object beam intensity. Crosstalk can be avoided by choosing a certain intensity ratio between the reference and the object beams.
\end{abstract}

Optical interconnections are of major importance in a variety of applications, covering neural networks, ${ }^{1}$ information storage, ${ }^{2,3}$ clock distribution, ${ }^{4}$ and optical computing. ${ }^{5}$ Storage of the interconnection pattern as holographic phase gratings in photorefractive crystals has been proposed as a solution to this problem. ${ }^{6,7}$ Some applications demand dynamic interconnections, i.e., the ability to turn an interconnection on and off. In other applications, such as neural networks, the interconnection needs to have an analog weight factor. We have studied an interconnect scheme based on angular multiplexing of holographic gratings in $\mathrm{Bi}_{12} \mathrm{SiO}_{20}$ (BSO) crystals. The first consideration is to choose a scheme featuring the desired requirements. When the scheme is chosen the problem of crosstalk has to be considered, i.e., whether a change in one interconnection affects the properties of other interconnections.

Our work is based upon the scheme proposed by Marrakchi et al. ${ }^{7}$ using phase modulations of the writing beams to implement the analog weight factor. In this letter, two important issues are addressed. First, crosstalk is investigated as a function of the intensity ratio between the reference and the object beams. Second, the effect on the crosstalk of varying the separation angle between the object beams is investigated.

The formation of phase gratings in photorefractive crystals can briefly be explained as follows. When two coherent laser beams interfere inside a photorefractive crystal, carriers are excited into the conduction band. Due to the charge transport mechanisms, i.e., drift and diffusion and retrapping of charges, a space-charge field builds up. This space-charge field affects the refractive index of the crystal via the Pockels' effect. Thus, the intensity distribution is converted into a modulation of the refractive index, i.e., a phase hologram is formed. ${ }^{8}$

The diffraction process in dynamic optical interconnects is usually based on the theory by Kogelnik. ${ }^{9}$ He shows that the diffraction efficiency as a function of the angular detun- ing $\Delta \theta$ falls off as a sinc $c^{2}$ function. It has its first null when the angular detuning from the Bragg angle is $\Delta \theta_{0}=2 \pi /\left(K_{G} L\right)$, where $K_{G}$ is the grating wave vector and $L$ the crystal thickness. Hence, two gratings with wave vectors of about equal magnitude can be resolved provided their angular separation exceeds $\Delta \theta_{0}$.

Three beams are incident on the crystal; one reference beam and two object beams with angular separation $\Delta \theta>\Delta \theta_{0}$. Furthermore, one of the beams suffer from a timevarying phase modulation $\psi(t)$. The three incident beams are written as

$$
\begin{aligned}
& u_{R}(\bar{r}, \omega)=u_{R} \exp \left\{i \bar{k}_{R} \cdot \bar{r}-i \omega_{0} t\right\} \\
& u_{1}(\bar{r}, \omega)=u_{1} \exp \left\{i \bar{k}_{1} \cdot \bar{r}-i \omega_{0} t\right\}, \\
& u_{2}(\bar{r}, \omega)=u_{2} \exp \left\{i \bar{k}_{2} \cdot \bar{r}-i\left\lceil\omega_{0} t+\psi(t)\right]\right\}
\end{aligned}
$$

The phase modulation is implemented by means of a piezomirror driven by a sinusoidal voltage. The resulting phase modulation of the reflected light for normal incidence has the form $\psi(t)=4 \pi \lambda^{-1} a_{0} \sin (\Omega t)=A_{0} \sin (\Omega t)$, where $\lambda$ is the wavelength, $a_{0}$ the elongation amplitude, and $\Omega$ the frequency of the mirror. Hence, the phase modulation depends on the driver voltage through $a_{0}$ and on the frequency $\Omega$. The intensity distribution in the crystal plane is

$$
\begin{aligned}
I= & I_{0}\left[1+m_{1} \cos \left(\left\{\bar{k}_{R}-\bar{k}_{1}\right\} \cdot \bar{r}\right)+m_{2}\left(\cos \left(\left\{\bar{k}_{R}-\bar{k}_{2}\right\} \cdot \bar{r}\right)\right.\right. \\
& \times \sum_{n=0}^{\infty} J_{n}\left(A_{0}\right) \cos (2 n \Omega t)+\sin \left(\left\{\bar{k}_{R}-\bar{k}_{2}\right\} \cdot \bar{r}\right) \\
& \left.\left.\times \sum_{n=0}^{\infty} J_{2 n+1}\left(A_{0}\right) \sin (\{2 n+1\} \Omega t)\right)\right]
\end{aligned}
$$




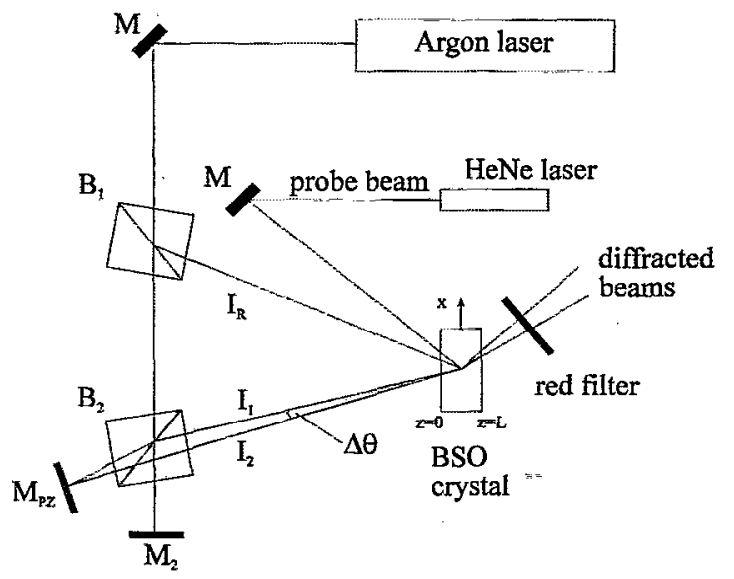

FIG. 1. The experimental setup.

where $J_{n}\left(A_{0}\right)$ is the Bessel function of order $n$ with argument $A_{0} . I_{0}=I_{R}+I_{1}+I_{2}$ is the total intensity, and $m_{i}$ $=2 \sqrt{I_{i} I_{R}} / I_{0}, i=1,2$, the modulation depth of the interference pattern. Equation (2) can be simplified because of the finite response time constant of the photorefractive crystal. Choosing $\Omega \gg \tau^{-1}$, the intensity distribution $I^{\prime}$ giving rise to the index modulation of the crystal can be found from Eq. (2) by neglecting all terms containing $n \Omega, n \geqslant 1$ :

$$
\begin{aligned}
I^{\prime}= & I_{0}\left[1+m_{1} \cos \left(\left(\bar{k}_{R}-\bar{k}_{1}\right) \cdot \bar{r}\right)\right. \\
& \left.+m_{2} J_{0}\left(A_{0}\right) \cos \left(\left(\bar{k}_{R}-\bar{k}_{2}\right) \cdot \bar{r}\right)\right] .
\end{aligned}
$$

The diffraction efficiency $\eta$ is proportional to the square of $m_{i}$ (for small values of $\eta$ and $m_{i}$ ).,9 Controlling the modulation depths yields the desired control over the interconnection pattern, and an interconnect is realized with a weight factor proportional to $m_{2}^{2} J_{0}^{2}\left(A_{0}\right)$. According to the definition of $m_{i}$, changing one interconnect does not affect the others, since the total intensity $I_{0}$ is unchanged. Hence, we may expect an interconnect scheme without crosstalk. Note, that if the beam $I_{2}$ is simply blocked, the interconnect $m_{1}$ will always be affected through the change in $I_{0}$.

The experimental investigation is concentrated on the crosstalk between two neighboring interconnections. The experimental setup is shown in Fig. 1. Two object beams $I_{1}$ and $I_{2}$, separated by an angle $\Delta \theta>\Delta \theta_{0}$, and a reference beam $I_{R}$ arc incident on a BSO sample. The sample is operated in the diffusion regime, i.e., no external voltage applied, in all the experiments reported here. The two object beams and the reference beam induce one grating $G_{1}$ due to the interference between $I_{R}$ and $I_{1}$, and another grating $G_{2}$ due to the interference between $I_{R}$ and $I_{2}$. The interference between the two object beams is neglected due to their small separation angle as the corresponding intensity distribution with a large fringe spacing is unable to induce a grating in the crystal. The phase modulation is implemented on $I_{2}$ with a piezomirror driven by a sine voltage with a frequency fulfilling $\Omega \gg \tau^{-1}$. When the phase shift is adjusted to a value that matches the first null of $J_{0}\left(A_{0}\right)$, the grating $G_{2}$ is selectively switched off according to Eq. (3). The plane writing beams originate from a single frequency $\mathrm{Ar}^{+}$-ion laser at wavelength $514.5 \mathrm{~nm}$. By

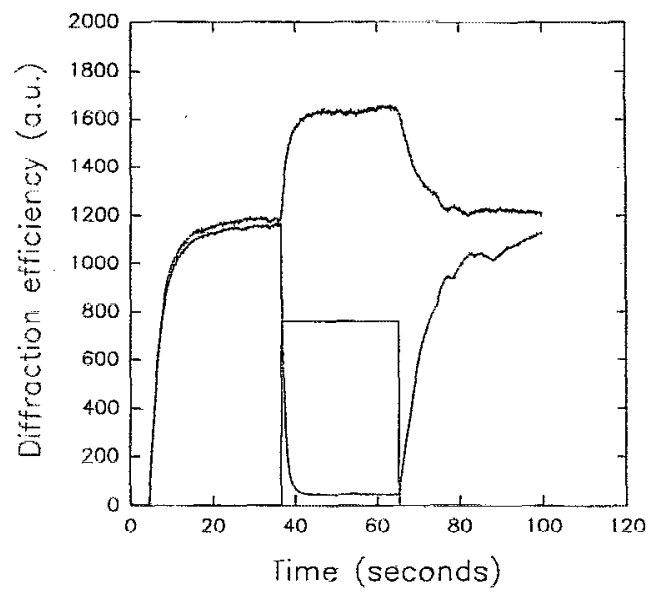

FIG. 2. Example of a single measurement in BSO with $m_{1}=0.51$ and $\Delta \theta$ -2.1 mrad. Note the strong increase in the diffraction efficiency in the remaining grating.

probing with a He-Ne laser at wavelength $632.8 \mathrm{~nm}$, the gratings are read out without interference from the probe beam. Adjusting the probe beam angle properly, two diffracted beams of equal strength are obtained in the output plane. These beams are detected by two photomultipliers with equal gain, and their output signals are sampled by a computer with an $A / D$ interface for subsequent analysis. Hence, this configuration allows simultaneous observation of the diffraction efficiency of both gratings.

A single measurement is obtained in the following manner, see Fig. 2. When a steady-state diffraction efficiency is reached with all three writing beams incident, the phase modulation of $I_{2}$ is turned on. This state is indicated by the drive voltage to the piezomirror being high. After steady state is reached again the change in diffraction efficiency of $I_{1}$ is measured. The modulation depth is then varied systematically by inserting neutral density filters in the beam paths and the crosstalk $\Delta \eta$ is measured for a fixed angle $\Delta \theta$. We define the crosstalk $\Delta \eta$ as $\Delta \eta=\left(\eta_{1, a}-\eta_{1, b}\right) / \eta_{1, b}$, where $\eta_{1, b}$ and $\eta_{1, a}$ represent the steady-state diffraction efficiencies of the nonphase modulated grating $G_{1}$ before and after modulation of $I_{2}$, respectively.

Figure 3(a) shows the crosstalk $\Delta \eta$ as a function of the modulation depth $m_{1}$ with $\Delta \theta=4.2 \mathrm{mrad}$ and fringe spacings of the two gratings $\Lambda_{1}=0.89 \mu \mathrm{m}$ and $\Lambda_{2}=0.88 \mu \mathrm{m}$, respectively. The two object beams are of equal strengths implying the same modulation depth of both gratings. The hollow circles correspond to the so-called weak reference situation and the filled circles to the strong reference situation. The two situations occur, since the intensity $I_{R}$ for fixed values of $m_{1}$ and $I_{1}$ is found from rewriting the definition of modulation depth $I_{R}^{2}+4 I_{1} I_{R}\left(1-m_{1}^{-2}\right)+4 I_{1}^{2}=0$ assuming $I_{1}=I_{2}$. Solving for $I_{R}$, we find values either larger than or smaller than the sum of the two object beams $I_{1}+I_{2}=2 I_{1}$. We define these solutions as the strong or weak reference situation. Figure 3(a) shows that crosstalk is a serious problem when the reference beam is weaker than the sum of the object beam intensities. The crosstalk is largest when the modulation depth is in the region $0.1 \leqslant m_{1} \leqslant 0.4$. In this region the 


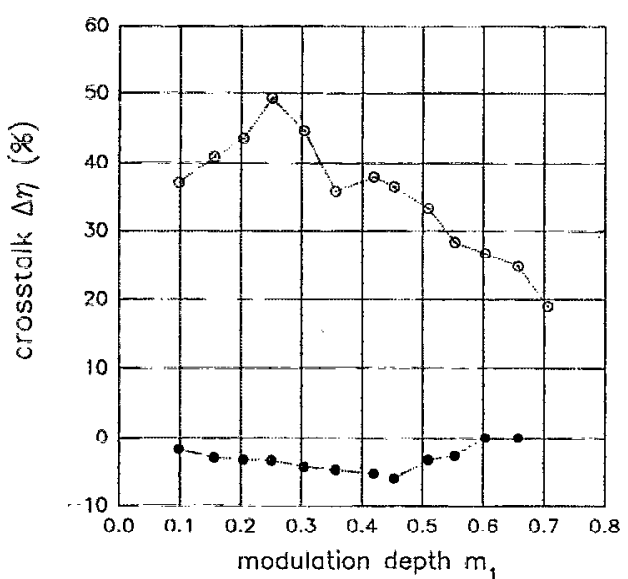

(a)

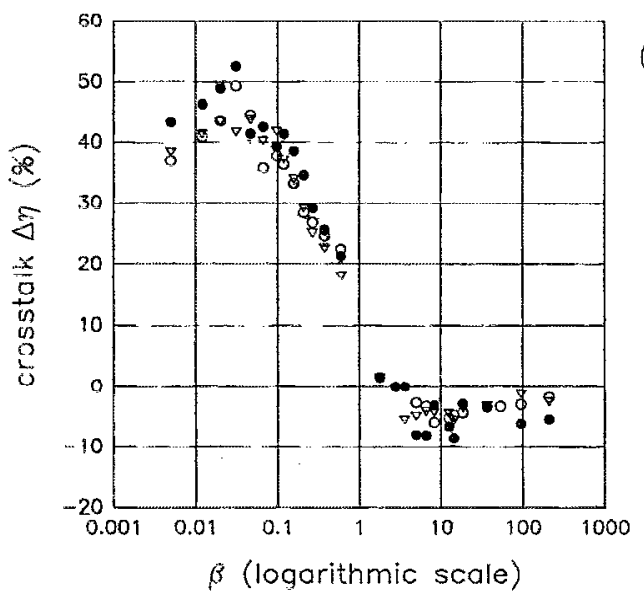

FIG. 3. (a) Crosstalk $\Delta \eta$ in $\%$ vs the modulation depth $m_{1}$. The fringe spacings are $\Lambda_{1}=0.89 \mu \mathrm{m}$ and $\Lambda_{2}=0.88 \mu \mathrm{m}$, and the separation angle is $\Delta \theta=4.2 \mathrm{mrad}$. Hollow circles correspond to the weak reference situation, filled circles correspond to strong reference beam situation. (b) Crosstalk $\Delta \eta$ $(\%)$ vs the intensity ratio $\beta$. Hollow circles correspond to $\Delta \theta=4.2 \mathrm{mrad}$, filled circles to $\Delta \theta=2.1 \mathrm{mrad}$, and hollow triangles to $\Delta \theta=5.2 \mathrm{mrad}$.

change in diffraction efficiency is close to $50 \%$. On the other hand, crosstalk is minimized if the reference beam is chosen to be stronger than the object beams with crosstalk in the range $0 \%$ to $-6 \%$. Note that the results obtained in Ref. 7 agree with the results in Fig. 3(a) only if $m_{1} \approx 0.65$ and strong reference. Comparing the experimental results with Eq. (3) and related remarks, however, it is evident that the simple description of grating formation in this interconnect scheme is incomplete. A more profound description of the grating formation is thus needed.

Furthermore, we have made two more series of measurements in BSO differing only by the choice of separation angle $\Delta \theta$. In the two following series, the separation angle is chosen to be 2.1 and $5.2 \mathrm{mrad}$, respectively. Plotting the results of these measurements yields similar results as shown in Fig. 3(a). From these measurements we conclude that the separation angle does not influence the crosstalk significantly. This fact implies that the crosstalk phenomenon is not resulting from off-Bragg diffraction properties. Furthermore, minimum crosstalk is observed when the reference beam is stronger than the sum of the object beams. Finally, it is not the modulation depth in the intensity pattern that determines the amount of crosstalk, but rather the intensity ratio of the reference intensity to the object beam intensities $\beta=I_{R} / I_{1}+I_{2}$. This is derived from the curves in the following way: the photorefractive crystal responds to the modulation depth thus yielding a certain value of the diffraction efficiency. A given modulation depth yields two different amounts of crosstalk although the diffraction efficiency is the same. Thus, it must be $\beta$ that controls the crosstalk, since the only difference of the two measurements is the reference beam intensity. We have obtained similar results in other BSO crystals.

The influence of the parameter $\beta$ is apparent in Fig. 3(b) where the crosstalk is plotted versus $\beta$ with $\Delta \theta=4.2 \mathrm{mrad}$ (filled circles), $\Delta \theta=2.1 \mathrm{mrad}$ (hollow circles), and $\Delta \theta=5.2$ mrad (hollow triangles). From this plot it is evident that the crosstalk is not dependent on the separation angle $\Delta \theta$ provided $\Delta \theta>\Delta \theta_{0}$. In an interconnect system, two main criteria have to be satisfied: high diffraction efficiency, i.e., high modulation depth, and low crosstalk. Figure 3(b) shows that crosstalk is low, if $2.5 \leqslant \beta \leqslant 4.5$. Assuming $I_{1}=I_{2}$, the modulation depth can be calculated from the expression $m=\sqrt{2 \beta} /(1+\beta)$, hence, the modulation depth is in the region $0.55 \leqslant m \leqslant 0.65$ yielding an acceptable diffraction efficiency. Actually, this is not far from the maximum obtainable modulation depth, which in the case of $I_{1}=I_{2}$ is $m$ $=1 / \sqrt{2} \approx 0.71$.

In conclusion, significant crosstalk in the range of $-10 \%-+50 \%$ between neighboring gratings has been observed using BSO crystals in dynamic optical interconnects. It is shown experimentally that the intensity ratio $\beta$ between the reference beam path and the object beam path controls the amount and sign of the crosstalk. Crosstalk is avoided by choosing $\beta$ properly. In the case of two object beams this ratio should be in the region $2.5 \leqslant \beta \leqslant 4.5$. If the Bragg selectivity is obeyed, the separation angle of the two object beams is unimportant with regard to the crosstalk.

One of the authors, Peter E. Andersen, was supported by the Danish Technical Research Council Grant No. 16-5047.

${ }^{1}$ D. Psaltis, D. Brady, and K. Wagner, Appl. Opt. 27, 1752 (1988).

${ }^{2}$ P. B. Berra, A. Ghafoor, M. Guizani, S. J. Marchinkowski, and P. A. Mitkas, Proc. IEEE 77, 1797 (1989).

${ }^{3}$ S. Hunter, F. Kiamilev, S. Esener, D. A. Parthenopoulos, and P. M. Rentzepis, Appl. Opt. 29, 2058 (1990).

${ }^{4}$ J. W. Goodman, F. I. Leonberger, S. Kung, and R. A. Athale, Proc. IEEE 72, 850 (1984).

${ }^{5}$ P. Yeh, A. E. Chiou, J. Hong, P. Beckwith, T. Chang, and M. Khoshnevisan, Opt. Eng. 28, 328 (1989).

${ }^{6}$ A. Chiou and P. Yeh, Appl. Opt. 29, 1111 (1990).

${ }^{7}$ A. Marrakchi, W. M. Hubbard, S. F. Habiby, and J. S. Patel, Opt. Eng. 29, 215 (1990).

${ }^{8}$ N. V. Kukhtarev, V. B. Markov, S. G. Odulov, M. S. Soskin, and V. L. Vinetskii, Ferroelectrics 22, 949 (1979).

${ }^{9}$ H. Kogelnik, Bell System Tech. J. 48, 2909 (1969). 